\title{
Contribuição da tutoria na formação acadêmica de graduandos em enfermagem
}

\author{
Contribution of tutoring in the academic education of undergraduates in nursing \\ Contribución de la tutoría en la formación académica de licenciaturas en enfermeira
}

Recebido: 07/02/2022 | Revisado: 15/02/2022 | Aceito: 23/02/2022 | Publicado: 04/03/2022

\author{
Matheus Tavares França da Silva \\ ORCID: https://orcid.org/0000-0003-4188-0115 \\ Universidade Estadual do Ceará, Brasil \\ E-mail: matheus.tavares@aluno.uece.br \\ Caroliny Cristine dos Santos Mendes \\ ORCID: https://orcid.org/0000-0002-8511-3471 \\ Universidade Estadual do Ceará, Brasil \\ E-mail: carolinymnds@gmail.com \\ Jordana Marjorie Barbosa do Nascimento \\ ORCID: https://orcid.org/0000-0003-0985-5155 \\ Universidade Estadual do Ceará, Brasil \\ E-mail: jordana.marjorie@gmail.com \\ Beatriz Davini Sales Rebouças \\ ORCID: https://orcid.org/0000-0001-7594-0898 \\ Universidade Estadual do Ceará, Brasil \\ E-mail: biadavini@gmail.com \\ Germana Pinheiro Correia Lima Sousa \\ ORCID: https://orcid.org/0000-0002-6550-1231 \\ Universidade Estadual do Ceará, Brasil \\ E-mail: germanapinheirosousa@gmail.com \\ Fernanda Abrantes de Oliveira Matias \\ ORCID: https://orcid.org/0000-0001-9008-0603 \\ Universidade Estadual do Ceará, Brasil \\ E-mail: nanda_abrantes98@hotmail.com \\ Rhanna Emanuela Fontenele Lima de Carvalho \\ ORCID: https://orcid.org/0000-0002-3406-9685 \\ Universidade Estadual do Ceará, Brasil \\ E-mail: rhanna.lima@uece.br
}

\begin{abstract}
Resumo
Objetivo: Verificar as contribuições das atividades de tutoria acadêmica desenvolvidas pelo PET Enfermagem, destinadas a acolher, acompanhar e orientar os calouros, assim como auxiliar na integração às atividades acadêmicas, científicas e culturais do curso e da universidade. Metodologia: Trata-se de uma pesquisa transversal de abordagem quantitativa, desenvolvida em uma instituição de ensino superior pública, localizada no estado do Ceará. Resultados: Contudo, destaca-se a continuidade da amostra no curso de graduação, especialmente entre aqueles que vivenciaram a experiência da tutoria acadêmica e a avaliação positiva dos discentes participantes, que reconhecem a influência da atividade na sua trajetória acadêmica, assim como demonstram-se satisfeitos com os assuntos abordados e recomendam a vivência da tutoria. Conclusão: Atingindo, dessa forma, os objetivos estabelecidos pelos pesquisadores de contribuir com o desenvolvimento acadêmico, estimulando e conscientizando os recém ingressos a rascunhar, gradualmente, as trajetórias de vida e carreira que querem construir.
\end{abstract}

Palavras-chave: Tutoria acadêmica; Enfermagem; Desempenho acadêmico; Ensino.

\begin{abstract}
Objective: To verify the contributions of the academic tutoring activities developed by PET Nursing, aimed at welcoming, monitoring and guiding the freshmen, as well as assisting in the integration of the academic, scientific and cultural activities of the course and the university. Methodology: This is a cross-sectional research with a quantitative approach, developed in a public higher education institution, located in the state of Ceará. Results: However, the continuity of the sample in the undergraduate course is highlighted, especially among those who have experienced the academic tutoring and the positive evaluation of the participating students, who recognize the influence of the activity on their academic trajectory, as well as demonstrate satisfied with the topics covered and recommend the experience of tutoring. Conclusion: Achieving, in this way, the objectives established by the researchers of contributing to academic development, stimulating and raising awareness among newcomers to gradually sketch the life and career paths they want to build.
\end{abstract}

Keywords: Academic tutoring; Nursing; Academic achievement; Teaching. 


\begin{abstract}
Resumen
Objetivo: Verificar las contribuciones de las actividades de tutoría académica desarrolladas por el PET Enfermería, con el objetivo de acoger, acompañar y orientar a los ingresantes, así como auxiliar en su integración a las actividades académicas, científicas y culturales del curso y de la universidad. Metodología: Se trata de una investigación transversal con enfoque cuantitativo, desarrollada en una institución pública de educación superior, ubicada en el estado de Ceará. Resultados: Sin embargo, se destaca la continuidad de la muestra en el curso de pregrado, especialmente entre quienes han experimentado la tutoría académica y la evaluación positiva de los estudiantes participantes, quienes reconocen la influencia de la actividad en su trayectoria académica, así como demuestran satisfecho con los temas tratados y recomendar la experiencia de tutoría. Conclusión: Logrando, de esta forma, los objetivos planteados por los investigadores de contribuir al desarrollo académico, estimulando y sensibilizando a los recién llegados para trazar paulatinamente los caminos de vida y carrera que quieren construir.
\end{abstract}

Palabras clave: Tutoría académica; Enfermería; Logro académico; Enseñando.

\title{
1. Introdução
}

Ao ingressar no ensino superior o estudante enfrenta um processo de transição e adaptação que resulta em uma série de sentimentos, dentre eles alívio das tensões geradas no período do vestibular, insegurança, ansiedade, felicidade, tensão e medo.

As vivências experienciadas pelo estudante no primeiro ano da faculdade caracterizam-se como cruciais para persistência no ensino superior e para o êxito acadêmico. Soares et al. (2018) afirmam que as expectativas dos estudantes nos primeiros anos universitários estão correlacionadas com as suas características pessoais, formação de amizades e o bom convívio social, preparação para o mercado de trabalho, oportunidade de reconhecimento e crescimento profissional, acesso a boa infraestrutura e a professores qualificados pertencentes ao colegiado do seu curso na instituição de ensino.

Acredita-se que um desafio nesse processo de transição compreende a adaptação ao novo, em alguns casos, acomodação à nova cidade; aos novos colegas; a nova realidade e ao novo sistema educacional, bem diferente do Ensino Médio (Oliveira et al., 2015). Nesse sentido, o novo ambiente exige do jovem bastante autonomia na aprendizagem, na administração do tempo e na definição de estratégias para o estudo. Segundo Oliveira et al., (2016), o ingresso no ensino superior é geralmente associado a acontecimentos singulares na vida dos estudantes, sendo permeado por modificações que requerem um esforço de ajustamento no indivíduo.

Diante desse cenário, os alunos bolsistas do Programa de Educação Tutorial (PET) de Enfermagem da Universidade Estadual do Ceará, desenvolveram um projeto de tutoria acadêmica como estratégia para facilitar a adaptação dos calouros ao ambiente acadêmico e contribuir com a permanência deste na universidade. De acordo com Alpes et al., (2018), a tutoria promove um ambiente propício para trocas de experiências e discussões sobre temas que envolvem o pessoal, profissional e social dentro e fora da Universidade, constituindo um momento de alívio para os estudantes diante das pressões características dessa fase da vida universitária.

Com a finalidade de abordar as contribuições da tutoria acadêmica para o estudante recém-ingresso no meio universitário, questiona-se: "Quais as contribuições das atividades de tutoria acadêmica desenvolvidas pelo PET-Enfermagem para universitários recém ingressos na universidade?".

Neste contexto, o presente estudo teve como objetivo verificar as contribuições das atividades de tutoria acadêmica desenvolvidas pelo PET Enfermagem, destinadas a acolher, acompanhar e orientar os calouros, assim como auxiliar na integração às atividades acadêmicas, científicas e culturais do curso e da universidade.

\section{Metodologia}

Trata-se de um estudo transversal analítico com abordagem quantitativa. Este é um método de pesquisa observacional que tem como objetivo analisar dados coletados a partir de tempo e de uma população pré-definidos (Aragão, 2011), 
desenvolvida em uma instituição de ensino superior pública, localizada no estado do Ceará. Trata-se de uma instituição multicampi que oferece 68 cursos de graduação presenciais das diversas áreas do conhecimento. O curso de graduação em Enfermagem é composto por nove semestres totalizando 304 créditos de disciplinas obrigatórias e 5.304 horas curriculares.

A população do estudo contemplou os acadêmicos de Enfermagem que ingressaram na universidade a partir da primeira edição do projeto até o presente momento, abrangendo as turmas de 2016.2 a 2019.2, totalizando 280 alunos, destes 95 acadêmicos aceitaram participar da pesquisa e compuseram a amostra.

A amostra foi constituída por sujeitos que atendiam aos seguintes critérios de inclusão: graduandos do curso de Enfermagem em situação de matrícula regular, que participaram de pelo menos $75 \%$ da carga horária da tutoria acadêmica. Excluiu-se os indivíduos que possuíam idade inferior a 18 anos.

O DesPErT consiste em uma modalidade de tutoria acadêmica, desenvolvida pelos 12 alunos bolsistas que compõem o Programa de Educação Tutorial (PET) de Enfermagem da Universidade Estadual do Ceará. O projeto possui como objetivo, auxiliar os recém ingressos do curso no processo de adaptação ao ambiente acadêmico. Os tutores acompanham os calouros durante todo o primeiro semestre da graduação, para isso os 40 calouros são divididos em 6 grupos de até 7 alunos, no qual cada grupo é acompanhado por 2 tutores. A participação na tutoria não é obrigatória e os alunos têm a liberdade de montar seus próprios grupos.

Por se tratar de um curso de graduação com regime integral, as reuniões são realizadas com frequência mensal e durante os intervalos das aulas, tendo aproximadamente $1 \mathrm{~h}$ e 30 minutos de duração. Nos encontros são acolhidas as demandas trazidas pelos tutorandos e são abordadas temáticas de relevância acadêmica, para situá-los nesse novo ambiente. Ao todo, durante cada semestre, são realizadas cinco reuniões com cada turma de calouros. Cada encontro contempla um objetivo específico voltado a cada grupo de alunos que estão ingressando na universidade, como mostra o Quadro 1 a seguir.

Quadro 1 - Temas abordados em cada reunião do DesPErT.

\begin{tabular}{|c|c|l|}
\hline Encontros & Temáticas & \multicolumn{1}{c|}{ Objetivos } \\
\hline $1^{\mathrm{a}}$ & Estresse Universitário & $\begin{array}{l}\text { Roda de conversa a respeito da saúde mental e da adequação ao novo } \\
\text { ambiente que é a universidade. }\end{array}$ \\
\hline $2^{\mathrm{a}}$ & Estratégias de Estudo & $\begin{array}{l}\text { Orientar calouros no que tange à estratégia de aprendizagem e } \\
\text { administração do tempo, de modo que percebam a responsabilidade da } \\
\text { organização pessoal na rotina de estudo. }\end{array}$ \\
\hline $3^{\mathrm{a}}$ & Eventos Científicos & $\begin{array}{l}\text { Mostrar a importância dos eventos científicos para a graduação, } \\
\text { ademais é explorado a diferença entre cada tipo de evento e como criar } \\
\text { uma apresentação coerente. }\end{array}$ \\
\hline $4^{\mathrm{a}}$ & Currículo Lattes & $\begin{array}{l}\text { Auxiliar na construção do currículo lattes e como utilizar a plataforma } \\
\text { da forma adequada. }\end{array}$ \\
\hline $5^{\mathrm{a}}$ & Encerramento & \multicolumn{1}{c}{ Avaliação dos encontros } \\
\hline
\end{tabular}

Fonte: Autores.

A coleta de dados ocorreu no período de janeiro a agosto de 2021, por meio de um questionário desenvolvido pelos pesquisadores contendo 10 questões, sendo todas objetivas. As questões são relacionadas a importância da tutoria acadêmica para o desenvolvimento do aluno dentro da universidade.

O questionário foi aplicado por meio de uma ferramenta on-line chamada Google Forms, na qual é possível criar formulários com links compartilháveis. Foram contatados 280 alunos, os quais receberam o link do formulário por e-mail e o Termo de Consentimento Livre e Esclarecido - TCLE, de acordo com a Resolução No 466/2012. Os participantes possuíam a 
liberdade de participar ou não do estudo, no qual foi ressaltado que a recusa não traria prejuízos em sua relação com o pesquisador ou com a instituição, tendo assim sua identidade preservada.

A organização dos dados provenientes do questionário deu-se a partir da tabulação destes no programa Microsoft Office Excel 2016, os quais foram sistematizados e, posteriormente, os achados mais relevantes divididos em categorias, buscando uma melhor organização para compreensão dos dados avaliados. Para o processamento dos dados foi utilizado a estatística descritiva por meio do software SPSS (Statistical Package for the Social Sciences), versão 20.0.

A pesquisa foi submetida e aprovada pelo Comitê de Ética da Universidade Estadual do Ceará com seguinte parecer, CAEE: 66645717.6.0000.5534.

\section{Resultados}

Os dados foram coletados com 95 acadêmicos das turmas de enfermagem da UECE que ingressaram entre os anos de 2016.2 a 2019.1 e que aceitaram participar da pesquisa. Vale ressaltar que dessa amostra 66,32\% vivenciaram o DesPErT. Entre os participantes do estudo, o semestre 2018.2 foi o que teve uma participação maior de alunos $(13,68 \%)$ se comparado aos demais semestres, sendo: 12,63\% do semestre 2016.2 participou do DesPErT; 8,42\% no semestre 2017.1; 6,31\% no semestre 2017.2 e 10,53\% no semestre 2019.1.

A partir disso, a porcentagem de $9,47 \%$ dos alunos encontra-se no segundo semestre, 17,89\% no terceiro semestre e igualmente no quarto semestre. Já $11,58 \%$ dos participantes estão no quinto semestre, $4,21 \%$ no sexto e $17,89 \%$ no sétimo semestre. Por fim, 15,79\% no oitavo semestre e 3,15\% no nono semestre. Ressalta-se que 96,84\% destes continuam cursando enfermagem na UECE. Ao serem indagados sobre a influência do DesPErT na vida acadêmica, $90,32 \%$ dos graduandos responderam que o projeto influenciou positivamente ao longo da graduação de Enfermagem.

Os alunos do curso de enfermagem participantes do DesPErT, quando indagados sobre a satisfação em participar dos encontros realizados no DesPErT, 93,55\% responderam que se sentiram satisfeito com a metodologia abordada. Ao mesmo tempo, 96,77\% dos participantes recomendaram o projeto para os demais (Tabela 1).

Tabela 1 - Avaliação do DesPErT pelos Discentes. Fortaleza, CE, Brasil, 2021.

\begin{tabular}{lcc}
\hline Itens & Sim & Não \\
& $\mathrm{N}(\%)$ & $\mathrm{N}(\%)$ \\
\hline O DesPErT influenciou na sua vida acadêmica? & $57(90,32)$ & $6(9,67)$ \\
Ficou satisfeito com os encontros do DesPErT? & $59(93,55)$ & $4(6,45)$ \\
Recomendaria o DesPErT para outros alunos? & $61(96,77)$ & $2(3,22)$ \\
\hline
\end{tabular}

Fonte: Autores.

A importância dos encontros realizados pelo DesPErT também foi questionada aos discentes. Diante disso, nas respostas avaliou-se os seguintes quesitos: 69,2\% afirmaram que o DesPErT auxiliou na adaptação ao ambiente acadêmico; $60 \%$ se beneficiaram da criação e atualização do currículo lattes; $58,4 \%$ informaram o engajamento nos projetos da universidade; $50,7 \%$ na participação de eventos; $41,5 \%$ que foi importante para auxiliar o estresse causado pela vida acadêmica e igualmente para construir um cronograma de estudo. Assim, os graduandos escolheram os itens que representavam a principal importância do projeto desenvolvido pelo grupo PET.

A seguir, a Tabela 2 especifica as atividades nas quais o DesPErT influenciou positivamente ou contribuiu para que os 
calouros participassem durante a jornada acadêmica, como a participação em grupos de pesquisa, em bolsa estudantil, centro acadêmico e estágios extracurricular.

Tabela 2 - Distribuição dos discentes do DesPErT na participação de atividades extracurriculares. Fortaleza, CE, Brasil, 2021.

\begin{tabular}{|c|c|c|c|}
\hline \multirow{2}{*}{ Variáveis } & \multicolumn{2}{|c|}{ DesPErT } & \multirow{2}{*}{$\begin{array}{c}\text { Total } \\
\mathbf{N}(\%)\end{array}$} \\
\hline & $\begin{array}{c}\text { Sim } \\
\mathbf{N}(\%)\end{array}$ & $\begin{array}{c}\text { Não } \\
\text { N (\%) }\end{array}$ & \\
\hline \multicolumn{4}{|l|}{ Grupo de Pesquisa } \\
\hline Sim & $50(79,3)$ & $25(78,1)$ & $75(78,9)$ \\
\hline Não & $13(20,6)$ & $7(21,8)$ & $20(21,0)$ \\
\hline \multicolumn{4}{|l|}{ Bolsista de Programas } \\
\hline Sim & $48(76,1)$ & $28(87,5)$ & $76(80)$ \\
\hline Não & $15(23,8)$ & $4(12,5)$ & $19(20)$ \\
\hline \multicolumn{4}{|l|}{ Centro Acadêmico } \\
\hline Sim & $5(8,6)$ & $5(15,6)$ & $10(10,5)$ \\
\hline Não & $58(92)$ & $27(84,3)$ & $85(84,4)$ \\
\hline \multicolumn{4}{|l|}{ Liga Acadêmica } \\
\hline Sim & $25(39,6)$ & $8(25)$ & $33(34,7)$ \\
\hline Não & $38(60.3)$ & $24(75)$ & $62(65,2)$ \\
\hline \multicolumn{4}{|c|}{ Estágio Extracurricular } \\
\hline Sim & $1(1,5)$ & $2(6,25)$ & $3(3,1)$ \\
\hline Não & $62(98,4)$ & $30(93,7)$ & $92(98,9)$ \\
\hline
\end{tabular}

Fonte: Autores.

Assim, a Tabela 2 mostra uma predileção dos discentes em algumas atividades extracurriculares, como por exemplo a pesquisa, devido à grande participação dos alunos nos grupos. Destaca-se também, a procura por bolsas acadêmicas, que podem se distribuir em iniciação científica, extensão universitária, monitoria acadêmica, programa de educação tutorial, programa de educação pelo trabalho e bolsas de estudo e permanência universitária. A seguir, a Tabela 3 especifica a participação dos discentes em cada atividade. 
Tabela 3 - Participação dos Discentes do DesPErT em bolsas acadêmicas. Fortaleza, CE, Brasil, 2021.

\begin{tabular}{|c|c|c|c|}
\hline \multirow{2}{*}{ Variáveis } & \multicolumn{2}{|c|}{ DesPErT } & \multirow{2}{*}{$\begin{array}{l}\text { Total } \\
\text { N }(\%)\end{array}$} \\
\hline & $\begin{array}{c}\text { Sim } \\
\mathbf{N}(\%)\end{array}$ & $\begin{array}{c}\text { Não } \\
\mathbf{N}(\%)\end{array}$ & \\
\hline \multicolumn{4}{|l|}{ Iniciação Científica } \\
\hline Sim & $24(38 \%)$ & $16(50 \%)$ & $40(42,1 \%)$ \\
\hline Não & $39(61 \%)$ & $16(50 \%)$ & $55(57,8 \%)$ \\
\hline \multicolumn{4}{|l|}{ Extensão Universitária } \\
\hline Sim & $15(23,8 \%)$ & $12(37,5 \%)$ & $27(26,3 \%)$ \\
\hline Não & $48(76,1 \%)$ & $20(62,5 \%)$ & $68(71,5 \%)$ \\
\hline \multicolumn{4}{|l|}{ Monitoria Acadêmica } \\
\hline Sim & $24(38 \%)$ & $13(40,6 \%)$ & $37(38,9 \%)$ \\
\hline Não & $39(61,9 \%)$ & $19(59,3 \%)$ & $58(61 \%)$ \\
\hline \multicolumn{4}{|c|}{ Programa de Educação Tutorial } \\
\hline Sim & $9(14,2 \%)$ & $4(12,5 \%)$ & $13(13,6 \%)$ \\
\hline Não & $54(85,7 \%)$ & $28(87,5 \%)$ & $82(86,3 \%)$ \\
\hline \multicolumn{4}{|c|}{ Programa de Educação pelo Trabalho } \\
\hline Sim & $4(6,3 \%)$ & $2(6,2 \%)$ & $6(6,3 \%)$ \\
\hline Não & $59(93,6 \%)$ & $30(93,7 \%)$ & $89(93,6 \%)$ \\
\hline \multicolumn{4}{|c|}{$\begin{array}{l}\text { Bolsas de Estudo e Permanência } \\
\text { Universitária }\end{array}$} \\
\hline Sim & $1(1,5 \%)$ & $3(9,3 \%)$ & $4(4,2 \%)$ \\
\hline Não & $62(98,4 \%)$ & $29(90,6 \%)$ & $91(95,7 \%)$ \\
\hline
\end{tabular}

Fonte: Autores.

Quanto à participação dos discentes em bolsas acadêmicas, destaca-se o engajamento dos alunos nas bolsas de iniciação científica e nas monitorias acadêmicas. Ademais, é possível observar que demais tiveram uma maior predileção pela extensão universitária.

Já em relação àqueles que não participaram do DesPErT, percebe-se uma menor participação em extensão universitária e monitoria acadêmica quando comparados aos que estiveram ativos na tutoria acadêmica. Assim, evidencia-se a contribuição da tutoria na carreira acadêmica dos graduandos em enfermagem.

\section{Discussão}

Diante dos dados apresentados, destaca-se a maior participação dos semestres iniciais na pesquisa, fato este que pode estar relacionado ao maior tempo de permanência destes na universidade, visto que os semestres finais se encontram inseridos nos campos de estágios. Ressalta-se também que o projeto foi bem avaliado pelos discentes participantes, que reconhecem a 
influência da atividade na sua trajetória acadêmica, assim como demonstram-se satisfeitos com os assuntos abordados e recomendam a vivência da tutoria.

Além disso, observa-se a continuidade da amostra no curso de graduação, especialmente entre aqueles que vivenciaram a experiência da tutoria acadêmica. Esse dado é de significativa notoriedade, visto que as perdas de estudantes que iniciam, mas não terminam seus cursos são desperdícios sociais, acadêmicos e econômicos. No setor público, são recursos públicos investidos sem o devido retorno. Nessas condições, cada vaga inativa em uma universidade pública é uma fonte de ociosidade de professores, funcionários, equipamentos e espaço físico, representando assim, uma perda profissional, econômica e social (Costa et al, 2015).

Esse prejuízo socioeconômico, evidencia a necessidade das instituições de ensino superior oferecerem ajuda e suporte aos estudantes no processo de adaptação ao contexto universitário, no sentido de facilitar a obtenção de bons resultados em suas trajetórias acadêmicas, pessoais e profissionais.

É inconcebível continuar a aceitar o papel do ensino superior como um adicionador de conhecimentos teóricos e científicos. Espera-se, neste contexto, que as instituições de ensino superior sejam capazes de analisar, acompanhar e sanar as questões sociais e econômicas, identificando problemas e contribuindo com soluções que preparem os universitários a trabalhar e conviver corretamente na sociedade. O que implica fortalecer outros modelos de ensino-aprendizagem como a tutoria, e concebê-la como uma metodologia imprescindível para o ensino (Simão et al, 2016).

Nesse contexto, por meio da avaliação dos tutorandos, evidencia-se a contribuição do projeto na adaptação ao ambiente acadêmico e no engajamento nas atividades extracurriculares. O suporte proporcionado pelo projeto, reforça a relevância da atividade, uma vez que a transição para a vida universitária e a integração social e acadêmica é essencial para fortalecer a rede de apoio do estudante dentro da universidade.

A integração acadêmica refere-se ao sentimento de fazer parte do ambiente universitário, ao contexto e às demandas inerentes a este, que incluem a satisfação com o desenvolvimento pessoal a partir das atividades vivenciadas pelo estudante, a afinidade com o curso e a qualidade e apoio recebido de docentes. Essa integração pode influenciar na decisão de permanência ou abandono do curso, já que estudantes que se inserem desde o início de sua jornada acadêmica apresentam maiores índices de aproveitamento das oportunidades oferecidas pela instituição, tanto na formação profissional quanto no seu desenvolvimento psicossocial, comparado aos que enfrentam dificuldades nessa transição à universidade (Franzoi et al., 2020).

Evidenciando, a contribuição do DesPErT no engajamento dos calouros em projetos da universidade. A relevância desse achado encontra-se em consonância com um estudo que observou que aqueles envolvidos em pelo menos uma atividade extracurricular, relataram com maior frequência estarem satisfeitos com o curso de graduação, o que pode estar associado à maior identificação com a profissão escolhida, tendo em vista as oportunidades de desenvolvimento no ambiente de formação (Nogario et al, 2016).

Atingindo, dessa forma, os objetivos estabelecidos pelos pesquisadores de contribuir com o desenvolvimento acadêmico e com a permanência na graduação, estimulando e conscientizando os recém ingressos a rascunhar, gradualmente, as trajetórias de vida/carreira que querem construir, por meio da inserção em atividades da universidade que facilitem concretizar tais projetos para a vida acadêmica e após a graduação.

Em relação ao envolvimento dos tutorandos em atividades acadêmicas, ressalta-se a participação em grupos de pesquisa e em bolsas universitárias. A relevância da evidência da participação dos tutorandos em grupos de pesquisa, encontra-se no fato de que o estudante tem, assim, oportunidade de experienciar o contato com as pesquisas realizadas entre os pósgraduandos, habituando-se às etapas da pesquisa e participando de alguma delas, o que lhe proporciona embasamento para $o$ desenvolvimento de novos projetos e investigações elaboradas individualmente. 
Dessa forma, abre espaço para discussão de projetos de pesquisa e de resultados entre professores, estudantes de graduação e pós-graduação. Devido a isso, a pesquisa constitui tema essencial na formação do enfermeiro, visando ao desenvolvimento da atitude investigativa no estudante, com repercussão favorável à aplicação dos conhecimentos científicos na futura prática profissional (Begui, 2020).

No entanto, sabe-se que uma formação acadêmica completa tem como requisitos além das atividades de pesquisa e ensino, a concomitante participação em atividades de extensão. Dentre essas, destacam-se as ligas acadêmicas. A participação em uma liga acadêmica promove diferentes visões ao aluno, permite abranger os horizontes para além da aprendizagem didática. $\mathrm{O}$ ganho de responsabilidades e a tomada de decisões contribuem para saber lidar com situações adversas e para a formação de perfis de liderança. Desse modo, as ligas acadêmicas acabam por se tornar instrumentos de exploração da autonomia, da criticidade, da criatividade e do comprometimento (Yang, 2019).

No que diz respeito às bolsas universitárias, destaca-se o envolvimento dos tutorandos na iniciação científica e na monitoria acadêmica, seja na condição de bolsista remunerado ou voluntário. Dentro dessa perspectiva, é notório que a inserção precoce do graduando na iniciação científica (IC) é um instrumento valioso para aprimorar qualidades desejadas em um profissional de nível superior, bem como para estimular e despertar a vocação para a pesquisa. Com isso, possibilita-se um direcionamento mais precoce de estudantes para a pós-graduação Stricto Sensu com menores taxas de evasão.

Essa assertiva vai de encontro ao resultado obtido em uma pesquisa, que evidenciou que o estímulo à iniciação científica por meio da institucionalização de programas contribuiu para a melhoria do rendimento acadêmico dos estudantes de odontologia, favorecendo uma completa e crítica formação profissional. A pesquisa científica proporciona ao estudante maior integração com a instituição e com os conteúdos, culminando em melhor desempenho acadêmico e formação mais abrangente (Nardini et al, 2019).

Por fim, destaca-se o engajamento dos acadêmicos tutorados em programas de monitorias acadêmicas de diversas disciplinas ao longo dos semestres letivos. A monitoria é uma ferramenta, por meio da qual os discentes têm a oportunidade de aprofundar conhecimentos, melhorar habilidades teóricas e práticas, solucionar dúvidas e fragilidades e ampliar o desenvolvimento pessoal, ético e profissional. Entende-se, assim, que o aluno-monitor vivencia uma aproximação com as atividades docentes, compreendendo o contexto do processo de educar, de forma que favoreça a ampliação do conhecimento e desperte-o para a docência (Cavalcante, 2021).

Dessa forma, é notória a influência do DesPErT na trajetória dos alunos que participaram do projeto, tanto no auxiliando na adaptação ao ambiente acadêmico e manejo do estresse desencadeado pelas mudanças quanto incentivando o engajamento nas atividades da universidade. Com relação às limitações do estudo, nota-se a dificuldade de conseguir atingir a amostra, pois muitos alunos já haviam concluído a graduação. Além disso, existem poucos estudos na comunidade científica sobre o tema abordado.

\section{Considerações Finais}

Evidenciou-se que a tutoria acadêmica foi uma estratégia exitosa, visto que 90,32\% dos alunos afirmaram que influenciou positivamente em suas vidas acadêmicas, especialmente na adaptação ao meio acadêmico e no engajamento nos projetos da universidade. Refletindo assim, na permanência universitária e na perspectiva de finalizar a graduação, pois nos últimos anos, 96,84\% dos alunos que participaram do DesPErT continuaram na graduação.

Além disso, $81,05 \%$ dos participantes da pesquisa relataram que se sentiram satisfeitos com a tutoria acadêmica e 70,53\% recomendaram a continuidade do projeto. Assim, enfatiza-se a importância da realização e divulgação de atividades semelhantes que permitam um ensinamento e um apoio mútuo, tanto para os bolsistas que organizam os encontros, quanto para os calouros que estão ingressando nesse momento novo e cheio de descobertas que é a universidade. 
Ademais com o objetivo de contribuir no acervo de pesquisas, faz-se necessário a construção de mais produções que validem a importância de orientar com empenho aos recém chegados às Universidades, levando em consideração suas dúvidas e anseios.

\section{Referências}

Alpes M. F., \& Wolf, A. E. (2018). Tutoria acadêmica (“mentoring”): Relato de experiência de um tutorado à tutor. Revista Extensão em Foco, (16), 90-98.

Aragão J. (2011). Introdução aos estudos quantitativos utilizados em pesquisas científicas. Revista Práxis, ano III, nº 6.

André, M. E. D. A., Pereira, R., Príncipe, L. M., \& Aranha, E. G. (2016). Tutoria acadêmica no mestrado profissional: um aprendizado compartilhado. Revista da FAEEBA - Educação e Contemporaneidade, 25 (47), 37-50.

Barbosa, M. M. F., Oliveira, M. C., Melo-Silva, L. L., \& Taveira, M. C. (2018). Delineamento e Avaliação de um Programa de Adaptação Acadêmica no Ensino Superior. Revista Brasileira de Orientação Profissional,19 (1), 61-74.

Begui, J. R., Guariente, M. H. D. de M., Garanhani, M. L., Carvalho, B. G. de, Ferrari, R. A. P., \& Galdino, M. J. Q. (2020). Pesquisa como princípio científico e educativo na formação do enfermeiro. Ciência, Cuidado E Saúde, 19, e:48380, 01-09.

Cavalcante, F. M. L, Menezes, A. C. V., Alves, D. G. S., \& Mendonça, G. M. M. (2021). Monitoria acadêmica em enfermagem: construindo conhecimentos através de metodologias ativas. Rev enferm UFPE on line, 15, e:244462, 01-10. https://periodicos.ufpe.br/revistas/revistaenfermagem/article/view/244462

Costa S. L., \& Dias, S. M. B. (2015). A permanência no ensino superior e as estratégias institucionais de enfrentamento da evasão. Jornal de políticas educacionais, 9 (17), e: 18, 51-60.

Costa, C. H. M., Chacon, L. D., Lima, A. B. L., Medeiros, R. S. P., \& Almeida, M. S. C. (2015). Perfil, motivos de ingresso e de evasão dos graduandos de Odontologia. Odontol. Clín.-Cient., 14 (3), 713-718.

Estevam, C., Basilio, A. J., Sticca, M. G., \& Versuti, F. M. (2018). Programa de tutoria por pares no ensino superior: Estudo de caso Artigo. Rev. bras. orientac. prof, 19 (2), 185-195.

Franzoi, M. A. H; \& Martins, G. Experiência de mentoring entre estudantes de graduação em enfermagem: reflexões e ressonâncias dialógicas. (2020). Interface - Comunicação, Saúde, Educação 24, e190772. https://doi.org/10.1590/Interface.190772

Hulley, S. B., Cummings, S. R., Browner, W. S., Grady, D. G, \& Newman, T. B. (2015). Delineando a Pesquisa Clínica (4a ed). ArtMed.

Nardini, E. F., Turssi, C. P., Silva, A. de S. F., \& Flório, F. M. Política de estímulo à iniciação científica: impacto no coeficiente de rendimento de graduandos em OdontologiaRevista da ABENO, 19, 1, 33-39.

Nota, L., Soresi, S., Ferrari, L., \& Ginevra, M. C. (2014). Vocational designing and career counseling in Europe: Challenges and new horizons. European Psychologist, 19(4), 248-259.

Oliveira, C. T., Santos, A. S., \& Dias, A. C. G. (2016). Expectativas de universitários sobre a universidade: sugestões para facilitar a adaptação acadêmica. Revista Brasileira de Orientação Profissional,17, (1), 43-53.

Oliveira, R. E. C., \& Moraes, A. (2015). Vivências acadêmicas e adaptação de estudantes de uma universidade pública federal do Estado do Paraná. $R$. Educ. Públ., 24 (57), 547-568.

Simão, A. M., Flores, M. S., \& Fernandes, S. R. G. (2016). Tutoria no ensino superior: concepções e práticas. Revista de Ciências da Educação, (7), 75-88. 2020 http://sisifo.ie.ulisboa.pt/index.php/sisifo/article/view/120

Soares, A. B., Leme, V. B. R., Gomes, G., Penha, A. P., Maia, F. A., Lima, C. A., Valadas, S., Almeida, L. S., \& Araújo, A. M. (2018). Expectativas acadêmicas de estudantes nos primeiros anos do Ensino Superior. Arq. bras. psicol. 70 (1), 206-223.

Vitória, M. I. C., Casartelli, A., Rigo, R. M., \& Costa, P. T. (2018). Engajamento acadêmico: desafios para a permanência do estudante na Educação Superior. Educação, 41 (2), 262-269.

Yang, G. Y. H., Braga, A. C. B., Hipólito, N. C., Sena, K., Vieira, T., Pessanha, C. G., Abrantes, F. G., Pereira, P. S., \& Corrêa, C. L. (2019). Liga de Anatomia Aplicada (LAA): as Múltiplas Perspectivas sobre Participar de uma Liga Acadêmica. Revista brasileira de educação médica, 43 (1), $80-86$. 